\title{
ANÁLISIS TÉRMICO, HIDROFORMILACIÓN DE CANFENO E HIDROGENACIÓN DE LIMONENO CON COMPLEJOS TRIFENILFOSFINA-RUTENIO(II) CONTENIENDO LIGANDOS N-HETEROCÍCLICOS
}

\section{THERMAL ANALYSIS, CAMPHENE HYDROFORMYLATION AND HYDROGENATION OF LIMONENE WITH TRIPHENYLPHOSPHINE COMPLEXES, RUTHENIUM (II) CONTAINING N-HETEROCYCLIC LIGANDS}

\author{
Fernando Cuenú Cabezas ${ }^{1}$ María Cristina Duque ${ }^{1}$, Alberto Bolaños Rivera ${ }^{2}$
}

\author{
${ }^{1}$ Laboratorio de Química Inorgánica y Catálisis, Programa de Química, Universidad del Quindío. \\ ${ }^{2}$ Departamento de Química, Universidad del Valle, Cali-Colombia \\ Fecha de recibido: Febrero 3 de 2010 \\ Fecha de aceptado: Junio 9 de 2010 \\ Correspondencia: Programa de Química, Universidad del Quindío, Avenida Bolívar calle 12 norte, Armenia- Quindío. Correo electrónico \\ fercuenu@uniquindio.edu.co
}

\section{RESUMEN}

En este trabajo reportamos los estudios térmicos de termogravimetría (TGA) y la actividad catalítica en la hidroformilación en fase homogénea de canfeno utilizando complejos del tipo [ $\mathrm{RuCl}_{2}\left(\mathrm{PPh}_{3}\right)_{2}(\mathrm{~N}$-het $\left.)\right]$, donde los $\mathrm{N}$-heterocíclicos son: 2-acetilpiridina (2-acpy); 3-acetilpiridina (3-acpy), 4-acetilpiridina (4-acpy), 2-aminopiridina (2-ampy); 3 aminopiridina (3-ampy) y 4aminopiridina (4-ampy). Los estudios de TGA muestran que los complejos presentan pérdidas de masa que fueron asignadas a la disociación de ligandos, la mayoría de los complejos disocian un ligando trifenilfosfina y un $N$-heterocíclico en la primera descomposición, para luego sufrir la descomposición total del complejo o la disociación de otro ligando. En la hidroformilación de canfeno La posición de los sustituyentes en el anillo de la piridina ejercen una notable influencia en la selectividad y actividad del catalizador, los sustituyentes donores de densidad de carga en el anillo piridínico y que se encuentran ubicados en posiciones orto inducen a que el complejo sea menos activo que cuando el complejo presenta sustituyentes piridínicos en posición para. El mismo fenómeno se observa con complejos en donde el anillo de la piridina se encuentra sustituido por grupos atractores de densidad de carga.

Palabras clave: hidroformilación, hidrogenación, rutenio(II), terpenos

\section{ABSTRACT}

We report thermal studies of thermogravimetry (TGA) and catalytic activity in the hydroformylation in homogeneous phase of camphene using complexes [RuCl2 (PPh3)2(N-het)2], where the N-heterocyclic are: 2- acetylpyridine (2-acpy); 3acetylpyridine (3-acpy), 4-acetylpyridine (4-acpy), 2-aminopyridine (2-Ampy) 3 aminopyridine (3-Ampy) and 4 - aminopyridine (4-Ampy). TGA studies showed that the complexes present mass loss which was assigned to the dissociation of ligands, most of the complexes dissociate a triphenylphosphine and a $\mathrm{N}$-heterocyclic ligand in the first decomposition, to then undergo the total decomposition of the complex or the dissociation of another ligand. In the camphene hydroformylation the position of the substituents on the pyridine ring exerts a remarkable influence on selectivity and catalyst activity, the substituents donors of charge density in the pyridine ring and that are located in ortho-positions induce the complex to be less active than when the complex presents pyridinic substituents in para-position. The same phenomenon is observed with complexes where the pyridine ring is substituted by groups of attractors of charge density.

Keywords: hydroformylation, hydrogenation, ruthenium (II), terpenes

\section{INTRODUCCIÓN}

Como una eficiente ruta para convertir olefinas y gas de síntesis $\left(\mathrm{CO}+\mathrm{H}_{2}\right)$ en los correspondientes aldehídos, la hidroformilación se ha convertido en uno de las más importantes aplicaciones de la catálisis homogénea en la industria $^{1-4}$. Las últimas investigaciones revelan que aldehídos ramificados opticamente puros son precursores para farmacéuticos y agroquímicos ${ }^{2}$. Debido a su importancia industrial, la hidroformilación ha recibido considerable atención tanto experimentalmente ${ }^{5-8}$ como teoricamente

Los complejos de rutenio están siendo muy utilizados en la hidrogenación e hidroformilación de terpenos los cuales son muy importantes para la industria de la perfumería y sabores artificiales. Los hidrocarburos terpenos se encuentran en los aceites esenciales, algunas veces en grandes cantidades. Ellos con frecuencia sirven como materiales de partida para la síntesis de fragancias y sabores, aunque contribuyen relativamente poco a las fragancias y aromas. Los terpenos son usados principalmente en la perfumería de pisos, shampoos, cremas etc. y para la reconstrucción de aceites esenciales. De los terpenos mono cíclicos, aquellos que poseen la estructura de para-mentadieno son los más importantes ${ }^{1}$.

El limoneno (1,8-p-mentadieno) se encuentra abundantemente en aceites esenciales presentes en la

Rev. Invest. Univ. Quindío (20): 18-23. Armenia - Colombia 
cáscara de frutas cítricas, llegando en algunos casos al 90\%, también se encuentra en especies de menta y coníferas. El limoneno se obtiene en grandes cantidades como un subproducto en la producción de jugo de naranja. Este compuesto es muy estable y barato y es usado en jabones y cosméticos ${ }^{2}$

Se conoce que las reacciones en fase homogénea están limitadas por las condiciones experimentales necesarias para mejorarlas ${ }^{11-13}$. Cuando la temperatura excede los $120-130^{\circ} \mathrm{C}$, la actividad de estos sistemas con frecuencia declina abruptamente y el rompimiento del enlace P-C en las fosfina se presenta, acompañada por la formación de clusters metálicos inactivos de alta nuclearidad, los cuales contienen ligandos fosfido ${ }^{14-18}$.

El análisis térmico de un compuesto es importante utilizarlo cuando se quiere conocer el comportamiento térmico de un determinado catalizador y en algunas ocasiones tiene alguna importancia en reacciones catalíticas cuando se quiere proponer un mecanismo en las reacciones catalíticas tales como la hidrogenación, hidroformilación, carbonilación, isomerización etc ${ }^{19}$.

En este trabajo se estudia la actividad catalítica en las reacciones de hidrogenación e hidroformilación en fase homogénea de limoneno y canfeno respectivamente, con complejos de rutenio del tipo $\left.\mathrm{RuCl}_{2}\left(\mathrm{PPh}_{3}\right)_{2}(\text { acpy })_{2}\right]$. Además se realiza el estudio del análisis termogravimétrico para cada uno de los complejos de rutenio

\section{PARTE EXPERIMENTAL}

\section{REACTIVOS}

Los reactivos empleados fueron obtenidos comercialmente de las firmas Aldrich y Sigma grado analítico y utilizados directamente; estos compuestos son los siguientes:

4-acetilpiridina (4-acpy), 3-acetilpiridina (3-acpy), 2acetilpiridina (2-acpy), trifenilfosfina $\left(\mathrm{PPh}_{3}\right)$, Canfeno, Limoneno, tricloruro de rutenio.

\section{SOLVENTES}

Se utilizó benceno como solvente, suministrado por casas comerciales (Merck y Sigma) y purificado con sodio y benzofenona por 8 horas.

\section{SÍNTESIS DE LOS COMPLEJOS}

La síntesis de los complejos se realizó siguiendo los métodos reportados en la literatura ${ }^{20-22}$, en donde la estereoquímica de los complejos del tipo $\left[\mathrm{RuCl}_{2}\left(\mathrm{PPh}_{3}\right)_{2}(\mathrm{~N}-\right.$ het $)_{2}$ ] es cis-dicloro-trans-bis(trifenilfosfina)-cis-bis( $\mathrm{N}$ het) ${ }^{5,6,7,11 \text {. }}$

\section{EQUIPOS}

Las reacciones catalíticas se realizaron en un reactor Parr de $125 \mathrm{~cm}^{3}$ y sus productos analizados en un cromatógrafo de gases Varian 3600 con detector de ionización de llama (FID), con una columna Carbowax 20M, 30 metros de longitud, diámetro interno 0,32 milímetros.

La cantidad de catalizador utilizada en cada una de las reacciones catalíticas es de $1,47 \times 10^{-5}$ moles y la de sustrato $1,47 \times 10^{-2}$ moles, en donde los sustratos utilizados son: canfeno, limoneno. El volumen de solvente utilizado es de $50 \mathrm{~mL}$

En las reacciones de hidroformilación de CANFENO y de hidrogenación de LIMONENO Las manipulaciones fueron realizadas utilizando vidrio de tipo Schlenk acopladas a una línea doble de vacío y argón. Los test catalíticos fueron realizados en una autoclave de acero inoxidable de $100 \mathrm{cc}$.

Cuando la reacción es la de hidrogenación de limoneno, el reactor se presurizó 20 atmósferas de hidrógeno y La cinética de la reacción se realizó tomando muestras del reactor a medida que la reacción se efectúa, con intervalos de tiempo los cuales dependían de la velocidad de la reacción. Cuando la reacción es la de hidroformilación de canfeno se presurizo con 90atm de hidrógeno y $45 \mathrm{~atm}$ de monóxido de carbono y el tiempo de reacción fue de 18 horas. En las dos reacciones la temperatura es de $100^{\circ} \mathrm{C}$

Los productos se analizaron en un cromatógrafo de gases Varian 3600 con detector de ionización de llama (FID), con una columna Carbowax 20M, 30 metros de longitud, diámetro interno 0,32 milímetros.

En las reacciones de hidroformilación de canfeno, los productos mayoritarios formados fueron, exclusivamente, un par de aldehídos diasteroisómeros, resultantes de la introducción de grupos formíl en el carbono metilénico de Canfeno.

Los estudios termogravimétricos de TGA se realizaron en un Shimadzu TGA-50H. Velocidad de calentamiento $10{ }^{\circ} \mathrm{C}$ por minuto, en una atmósfera de nitrógeno.

\section{RESULTADOS Y DISCUSIÓN}

\section{Análisis elemental}

El análisis elemental calculado es consistente con el experimental mostrando que la fórmula de los compuestos es la correcta (ver tabla 1.)

Rev. Invest. Univ. Quindío (20): 18- 23. Armenia - Colombia 
Tabla 1. Análisis elemental de los complejos del tipo $\left[R u C l_{2}\left(P P h_{3}\right)_{2}(\mathrm{~N}-h e t)_{2}\right]$

Calculado Experimental

\begin{tabular}{ccccccc}
\hline Compuesto & $\% \mathrm{C}$ & $\% \mathrm{H}$ & $\% \mathrm{~N}$ & $\% \mathrm{C}$ & $\% \mathrm{H}$ & $\% \mathrm{~N}$ \\
{$\left[\mathrm{RuCl}_{2}\left(\mathrm{PPh}_{3}\right)_{2}(4-\mathrm{acpy})_{2}\right]$} & 56,34 & 4,69 & 2,98 & 56,37 & 4,67 & 2,97 \\
{$\left[\mathrm{RuCl}_{2}\left(\mathrm{PPh}_{3}\right)_{2}(3-\mathrm{acpy})_{2}\right]$} & 56,34 & 4,69 & 2,98 & 56,38 & 4,71 & 3,12 \\
{$\left[\mathrm{RuCl}_{2}\left(\mathrm{PPh}_{3}\right)_{2}(2-\mathrm{acpy})_{2}\right]$} & 56,34 & 4,69 & 2,98 & 56,31 & 4,73 & 3,08 \\
\hline
\end{tabular}

\section{Análisis térmico TGA de los complejos del tipo} [RuCl2(PPh3)2(N-het)2]

Los resultados de los estudios térmicos TGA de los complejos se dan en la tabla 2 , en donde se observa se presentan pérdidas de masa.

Tabla 2. Estudios termogravimétricos de los complejos del tipo [RuCl2(PPh3)2(N-het)2]

\begin{tabular}{|c|c|c|}
\hline \multicolumn{3}{|c|}{$\mathrm{RuCl}_{2}\left(\mathrm{PPh}_{3}\right)_{2}(4-\mathrm{acpy})_{2}, \mathrm{~m}_{0}=3,454 \mathrm{mg}$} \\
\hline Temperatura ${ }^{\circ} \mathrm{C}$ & 291 & 487 \\
\hline$\Delta \mathrm{m},(\mathrm{mg})$ & $-1,379$ & $-1,225$ \\
\hline \multicolumn{3}{|c|}{$\mathrm{RuCl}_{2}\left(\mathrm{PPh}_{3}\right)_{2}(3-\mathrm{acpy})_{2}, \mathrm{~m}_{0}=3,191 \mathrm{mg}$} \\
\hline Temperatura ${ }^{\circ} \mathrm{C}$ & 274 & 474 \\
\hline$\Delta \mathrm{m},(\mathrm{mg})$ & $-1,391$ & $-1,037$ \\
\hline \multicolumn{3}{|c|}{$\mathrm{RuCl}_{2}\left(\mathrm{PPh}_{3}\right)_{2}(2-\mathrm{acpy})_{2}, \mathrm{~m}_{0}=4,007 \mathrm{mg}$} \\
\hline Temperatura $^{\circ} \mathrm{C}$ & 297 & 513 \\
\hline$\Delta \mathrm{m}(\mathrm{mg})$ & 1,215 & $-0,679$ \\
\hline
\end{tabular}

Caracterización de las curvas TGA del complejo [RuCl2(PPh3)2(4-acpy)2]

La curva térmica TGA para el complejo muestra que el complejo sufre dos pérdidas de masa a $291^{\circ} \mathrm{C}$ y una segunda pérdida a $487^{\circ} \mathrm{C}$ (ver figura 1). La primera pérdida de masa es consistente con la disociación de una molécula de PPh3 y una de 4-acpy, el porcentaje de error calculado para esta disociación es del 0,5\%, lo cual corrobora nuestra interpretación. En la figura 2 se muestra la caracterización del espectro TGA del complejo. La especie tetracoordinada es estable hasta los $487^{\circ} \mathrm{C}$ en donde se presenta la segunda pérdida de masa, que no pudo ser caracterizada.

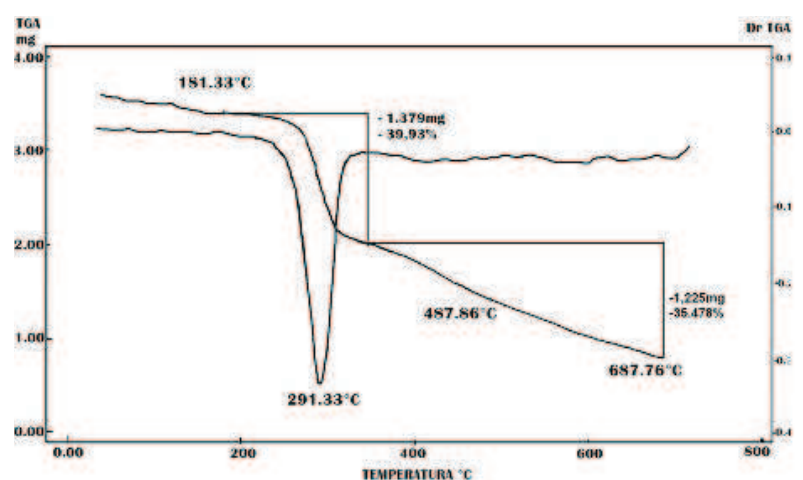

Figura 1. Termograma del complejo [RuCl2(PPh3)2(4-acpy)2

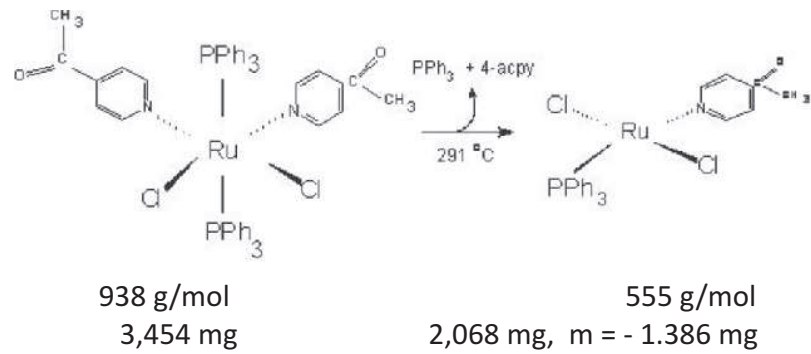

Figura 2 Caracterización de las curvas TGA del complejo [RuCl2(PPh3)2(4-acpy)2]

\section{Caracterización de las curvas del complejo [RuCl2(PPh3)2(3-acpy)2]}

El complejo sufre dos pérdidas de masa, las cuales han sido asignadas a la disociación de los ligandos. La primera pérdida de masa se presenta a 274 으, siendo consistente con la disociación de una trifenilfosfina y una molécula de 3-acetilpiridina. La segunda descomposición se presenta a 474 ㅇ $\mathrm{C}$ y es consistente con la disociación de la segunda molécula de trifenilfosfina. luego se presenta la descomposición total de la molécula (ver figura 3). 1 
<smiles></smiles>

$938 \mathrm{~g} / \mathrm{mol}$

$2 \quad 3,191 \mathrm{mg}$
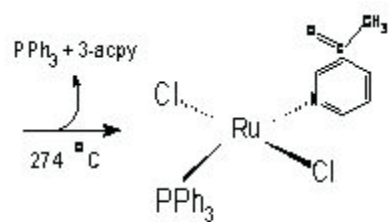

$555 \mathrm{~g} / \mathrm{mol}$ $1,8881 \mathrm{mg}, \mathrm{m}=-1,303 \mathrm{mg}$<smiles></smiles>

$555 \mathrm{~g} / \mathrm{mol}$ $1,8881 \mathrm{mg}$

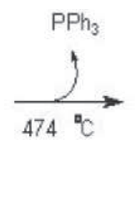

$0,9968 m g, m=-0,8913 m g$
Figura 3 Caracterización de las curvas TGA de [RuCl2(PPh3) 2(3-acpy)]

Caracterización de las curvas del complejo [RuCl2(PPh3)2(2-acpy)2]

La curva TGA del complejo (ver figura 4) muestra que se ocurren tres pérdidas de masa, a 297, 366 y 513 oC. La primera disociación es consistente con la pérdida de una molécula de $\mathrm{PPh} 3\left(297^{\circ} \mathrm{C}\right)$ y en la segunda disociación la de la otra molécula de PPh3. La tercera descomposición no pudo ser caracterizada (ver figura 5).

Puesto que, el grupo acetilo al estar en posición orto al nitrógeno unido al metal, puede generar la formación de un quelato al unirse al metal por medio del oxígeno, esta reacción es favorecida cuando se presentan sitios de coordinación vacantes, producidos por la disociación de uno de los ligandos. Formando nuevamente una especie estable de 18 electrones y además esta estabilidad puede ser aumentada porque se forma un anillo quelato de cinco miembros. Esta puede ser la explicación de el porque no se disocia una molécula de 2-acpy, como si ocurre en los complejos con la 4-acpy y 3 -acpy.

Lo anterior es importante en las reacciones catáliticas pues se puede indicar que este complejo presentará una menor actividad catálitica, porque los sustratos competirán con el grupo acetilo por el sitio de coordinación.

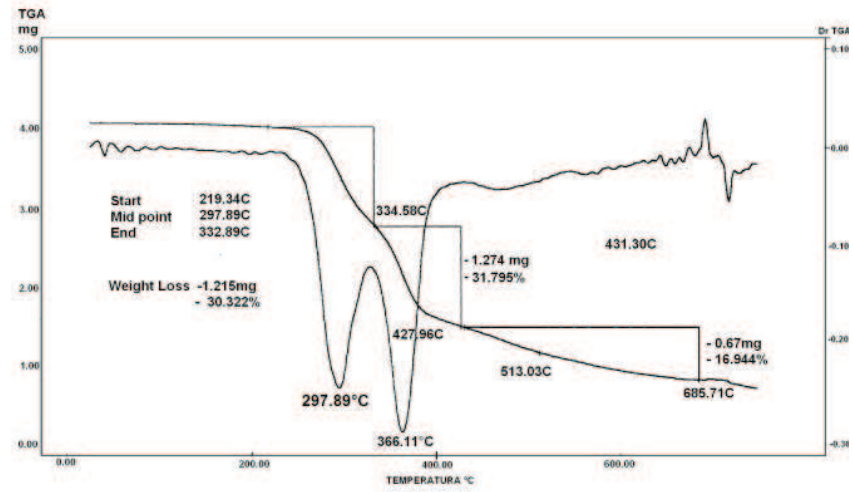

Figura 4. Termograma del complejo [RuCl2(PPh3)2(2-acpy)2

1

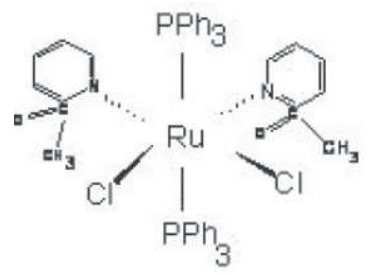

$938 \mathrm{~g} / \mathrm{mol}$

$2 \quad 4.007 \mathrm{mg}$

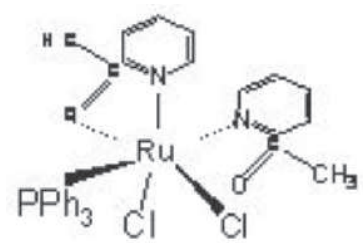

$676 \mathrm{~g} / \mathrm{mol}$

$2.8878 \mathrm{mg}$

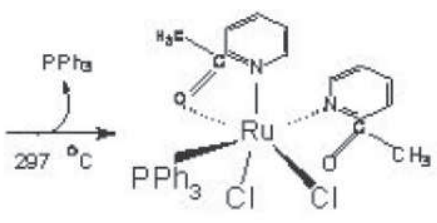

$676 \mathrm{~g} / \mathrm{mol}$

$2.8878 \mathrm{mg}, \mathrm{m}=-1,1192 \mathrm{mg}$
Figura 5 Caracterización de las curvas TGA de [RuCl2(PPh3)2(2acpy)2]
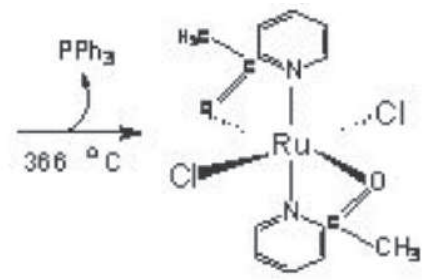

$414 \mathrm{~g} / \mathrm{mol}$

$1,7686 \mathrm{mg}, \mathrm{m}=-1,1192 \mathrm{mg}$

\section{HIDROGENACIÓN DE LIMONENO}

En la hidrogenación en fase homogénea de limoneno, se observa que se presentan tres productos de reacción con el complejo RuCl2(PPh3)2(2-acpy)2 (ver tabla 3), en donde dos de ellos son productos de la reacción de hidrogenación y el otro de la reacción de isomerización, (ver figura 6) Puesto que, el 2-p-menteno se obtiene cuando el 1-p-menteno ya se ha generado, se produce por la isomerización del 1-pmenteno. El Terpinoleno se produce a través de una reacción de isomerización del limoneno. Lo anterior nos indica que la selectividad hacia los productos de hidrogenación es del $91 \%$, en tanto que para los complejos 
con 4-apy y 3 -acpy es del 98 y $86 \%$ respectivamente.<smiles>C=C(C)C1CC=C(C)CC1</smiles>

Limoneno
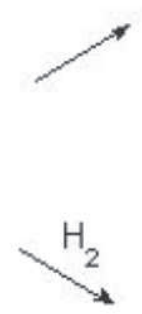<smiles>CC1=CCC(C(C)C)CC1</smiles>

1-p-menteno<smiles>CC1=CCC(=C(C)C)CC1</smiles>

Terpinoleno
Figura 6. Productos de la hidrogenación de Limoneno con [RuCl2(PPh3)2(2-acpy)2]

La tabla III, indica que el complejo con la 4-acpy es regioselectivo hacia la reacción de hidrogenación del doble enlace exo-cíclico (98\%), con un porcentaje de conversión del $83 \%$.

Tabla 3. Hidrogenación de limoneno con los complejos de Ru(II)

\begin{tabular}{|c|c|c|c|}
\hline \multicolumn{4}{|c|}{ \% de los productos de reacción } \\
\hline Limoneno & $1-p$-menteno & $2-p$-menteno & Terpinoleno \\
\hline \multicolumn{4}{|c|}{$\mathrm{RuCl}_{2}\left(\mathrm{PPh}_{3}\right)_{2}(2 \text {-acpy })_{2}$} \\
\hline 44,21 & 50,32 & 4.57 & 0.86 \\
\hline \multicolumn{4}{|c|}{$\mathrm{RuCl}_{2}\left(\mathrm{PPh}_{3}\right)_{2}(3-\mathrm{acpy})_{2}$} \\
\hline 0 & 75.7 & 12.28 & 0.0 \\
\hline \multicolumn{4}{|c|}{$\mathrm{RuCl}_{2}\left(\mathrm{PPh}_{3}\right)_{2}\left(4-\mathrm{acpy}_{2}\right.$} \\
\hline 15.69 & 83.4 & 0.91 & 0.0 \\
\hline
\end{tabular}

En la hidrogenación selectiva en fase homogénea de limoneno utilizando los catalizadores de rutenio anteriormente citados indican que, los factores estéricos y electrónicos influyen tanto en la actividad como en la selectividad del catalizador. Al variar las posiciones de los sustituyentes en el anillo de la piridina, se encontró que sustituyentes atractores de densidad electrónica en posición meta inducen a que la especie sea muy activa, generando una conversión del limoneno del 100 \%. Los impedimentos estéricos generados por los sustituyentes en posición orto en el anillo de la piridina inducen a que el complejo sea menos activo, lo anterior también puede darse por el bloqueo de los sitios de coordinación, lo cual impide un buen acercamiento de los sustratos. La selectividad por las reacciones de isomerización se favorecen por los factores electrónicos inducidos por estos grupos en posición orto.

Los estudios catalíticos muestran que los complejos son regioselectivos hacia la hidrogenación de dobles enlaces exo-cíclicos, indicando que los impedimentos estéricos en la molécula inducen a que la especie activa presente dificultades en hidrogenar dobles enlaces endo-cíclicos en donde uno de los carbonos es terciario.

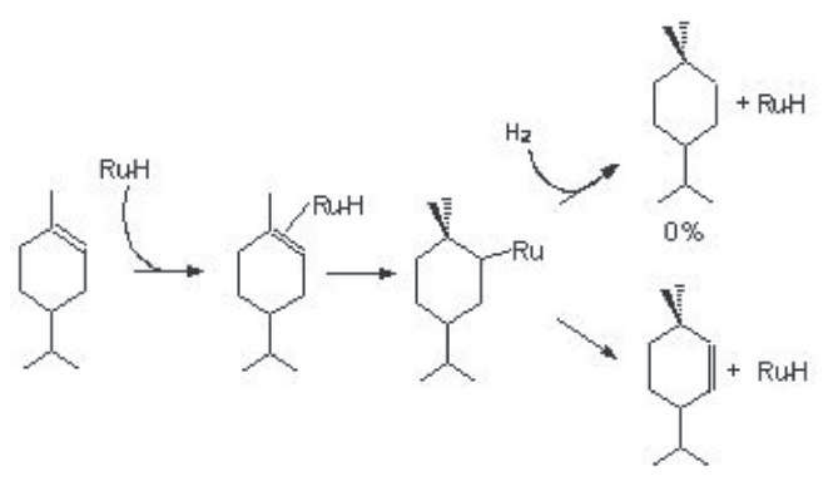

Figura 7. Isomerización del 1-p-menteno

$100 \%$

Para todos los complejos se observa que presentan poca selectividad hacia las reacciones de isomerización, por lo que la producción de terpinoleno es muy baja o no se presenta, lo cual puede ser explicado por los factores estéricos que inhiben las reacciones de eliminación de hidrogeno y los factores electrónicos favorecen las adiciones oxidativas de hidrógeno.

\section{HIDROFORMILACIÓN EN FASE HOMOGÉNEA DE CANFENO}

En la tabla 4 se presentan los resultados de actividad y selectividad para varios complejos de rutenio en la hidroformilación de Canfeno.

Todos los complejos estudiados se mostraron activos en la hidroformilación de Canfeno y no fueron observados los productos de isomerización del esqueleto del terpeno como ocurre cuando se emplea complejos de cobalto como catalizadores. 2 Los complejos con piridinas substituidas en las posiciones 2 y 3 son menos activos cuando son comparados con los que contienen piridinas substituidas en la posición 4 (ver tabla 3). La selectividad para el aldehído con menor tiempo de retención cromatográfico (CG Carbowax 20M), atribuida a la estructura 1, fue mayor para los complejos conteniendo piridinas substituidas en la posición 4. El inverso fue observado para las piridinas en las posiciones 2 y 3 . 
Tabla 4. Hidroformilación de Canfeno con los complejos del tipo RuCl2(PPh3)2(N-het)2

\begin{tabular}{ccccc}
\hline N-het. & $\begin{array}{c}\text { \%Conversión } \\
\text { del sustrato. }\end{array}$ & $\begin{array}{c}\text { Selectividad a la } \\
\text { hidroformilación }\end{array}$ & $\begin{array}{c}\text { Distribución de los } \\
\text { productos en porcentaje }\end{array}$ \\
\cline { 3 - 5 } & & & Aldehído 1 & Aldehído 2 \\
\hline 4-acpy & 99 & 100,00 & 56 & 42 \\
3-acpy & 55 & 100,00 & 21 & 34 \\
2-acpy & 57 & 100,00 & 24 & 30 \\
\hline
\end{tabular}

a. La distribución de los aldehídos puede ser inversa.

La hidroformilación de Canfeno con piridinas sustituidas por grupos atractores de densidad electrónica muestran que los complejos en donde el sustituyente se encuentra en posición orto o meta, son poco activos y poco selectivos, mientras que el complejo en donde el grupo atractor de carga se encuentra en posición para del anillo piridínico, es muy activo y muy selectivo hacia la producción de aldehídos.

La posición de los grupos unidos al anillo de la piridina ejercen una notable influencia en la selectividad y actividad del catalizador donores de densidad de carga.

En conclusión, determinamos que los complejos del tipo $\mathrm{RuCl} 2$ (PPh3)2(N-het)2 presentan una actividad promisoria para la hidroformilación de terpenos asociada a una elevada selectividad.

\section{AGRADECIMIENTOS}

A la Universidad del Quindío, a la Universidad del Valle, al profesor Eduardo Nicolau Dos Santos director del laboratorio de Catálisis y a la profesora Irene directora del Laboratorio de Análisis térmico de la Universidad Federal de Minas Gerais (Brasil).

\section{BIBLIOGRAFÍA}

1. Van leeuwen, P.W. N.M.; Claver, C. Rhodium Catalyzed hydroformylation; Kluwer Acacemic Publishers; Dordrecht, Netherlands, 2000. Falbe, J. New Shyntheses with Carbon Monoxide, Springer-Verlag; Berlin, 1980.

2. Torrent, M.; Solà, M.; Frenking, G. Chem. Rev. 2000, 100, 439.

3. Cornils, B.; Herrman, W. A. Applied Homogeneus Catalysis with organometallics Compounds, Wiley-VCH: Weinhein. 2000, Vol. 1.

4. Heck, R.F. Adv. Organomet. Chem. 1966, 4, 243.

5. Tominaga, K. I.; Sasaki, Y. J. Mol. Cat. A. 2004, 220, 159

6. Diéguez, M.; Pamies,O.; Claver, C. tetrahedron: asymmetry, 2004, 15, 2113; Clarke, M. L. Tetrahedron letters, 2004, 45, 4043.

7. Hegedus, C.; Madarász, J.; Gergely,I.; Szollosy, A.; Monsees, A.; Riermeier,T.; Bakos, J. tetrahedron: asymmetry, 2004, 15, 2507.

8. Herseczki, Z.; Gergely,l.; Szollosy, A.; Hegedus, C.; Bakos, J. tetrahedron: asymmetry, 2004, 15, 1673.

9. Niu. S; Hall, M.B. Chem. Eur J. 1998, 100, 353.

10. Huo,C.F.; Li, Y.W.; Beller, M.; Jiao, H. Organometallics, 2003, 22,4665.

11. Wender, I.; Pino, P. (eds.), Organyc Syntheses via Metal Carbonyls, Wiley, New York, 1977.

12. Wilkinson, G.; Stone, F.; Abel, E. (eds.), Comprehensive Organometallic Chemistry, Pergamos, Oxford, 1982.

13. Hegedus, L., Transition Metal in the synthesis of Complex Organic Molecules, University Science Books, Mill Valley, CA, 1994.

14. Garrou, P., Chem. Rev., 1985, 85, 171.

15. Frediani, P.; Bianchi, M.; Salvini, A.; Piacenti, F.; lanelli, S.; Nardelli, M. J. Chem. Soc., Dalton Trans. $1990,165$.

16. Frediani, P.; Bianchi, M.; Salvini, A.; Piacenti, F.; lanelli, S.; Nardelli, M. J. Chem. Soc., Dalton Trans. 1990, 1705.

17. Frediani, P.; Bianchi, M.; Salvini, A.; Piacenti, F.; lanelli, S.; Nardelli, M. J. Chem. Soc., Dalton Trans. 1990, 3663.

18. Frediani, P.; Bianchi, M.; Salvini, A. Guarducci, R., Carluccio C.; Piacenti, FJ. Organomet. Chem. 1995, $498,187$. 